\title{
DER NACHWEIS
}

VON

\section{SCHRIFTFÄLSCHUNGEN, BLUT, SPERMA Usw.}

UNTER BESONDERER BER ÜCKSICHTIGÚNG DER

\section{PHOTOGRAPHIE}




\section{DER NACHWEIS}

\section{rox \\ CHRIFTFÄLSCHUNGEN, BLUT, SPERMA usW.}

UNTER BESONDERER BERÜCKSICHTIGUNG DER

\section{PHOTOGRAPHIE}

MIT

EINEM ANHANGE ÜBER BRANDSTIFTUNGEN

$\mathbf{F} \ddot{\mathbf{U}} \mathbf{R}$

CHEMIKER, PHARMAZEUTEN, MEDIZINER, JURISTEN, POLIZEIORGANE USW.

VON

PROF. Dr. M. DENNSTEDT

DIREKTOR DES CHEMISCHEN STAATS-LABORATORIUMS IN HAMBURG

UN D

Dr. F. VOIGTL $\ddot{A} N D E R$

ASSISTENT AM CHEMISChEN STAATS-LABORATORIUM IN HAMBURG

MIT 97 ABBILDUNGEN EINSCHL. EINER FARBIGEN SPEKTRALTAFEL

Springer Fachmedien Wiesbaden $\mathrm{GmbH}$

1906 
Alle Rechte,

namentlich dasjenige der Utbersetzung in fremde Sprachen, vorbehalten.

Published January 1, 1906.

Privilege of Copyright in the United States reserved under the Act approved March 3,

(C) Springer Fachmedien Wiesbaden 1905

Ursprünglich erschienen bei Friedr. Vieweg \& Sohn, Braunschweig Germany 1905

Softcover reprint of the hardcover 1st edition 1905

ISBN 978-3-663-19845-1

ISBN 978-3-663-20182-3 (eBook)

DOI 10.1007/978-3-663-20182-3 


\section{O R R E D E.}

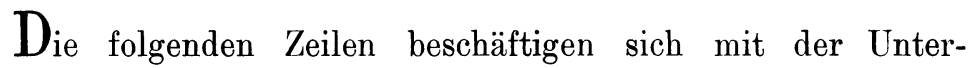
suchung von Schriftfälschungen, dem Nachweise von Blut und Sperma, soweit der Gerichtschemiker damit befaßt zu werden pflegt.

Hierbei sind die photographischen Verfahren in erster Linie behandelt und besonders hervorgehoben; man würde jedoch in der Annahme irren, daß die Photographie allein imstande ist, in den angeführten Untersuchungen den Chemiker in allen Fällen ans Ziel zu führen. Das ist durchaus nicht der Fall, und wenn auch z. B. bei manchen Urkundenfälschungen gerade die Photographie geeignet ist, Klarheit herbeizuführen, während die übrigen Methoden versagen, so sind doch auch die anderen physikalischen und chemischen Hilfsmittel dem Sachverständigen immer durchaus unentbehrlich.

Noch mehr zurück tritt die Photographie bei der Untersuchung verdächtiger Flecke von Blut und Sperma; hier zeigt sie weniger forschenden, als vielmehr registrierenden Charakter, indem sie das sicher festhält und aufbewahrt, was der Gerichtschemiker auf andere Weise, mit anderen Hilfsmitteln zu entdecken vermochte. Aber namentlich beim Blute verliert sie den ersten Charakter nicht ganz, besonders bei der Unterscheidung der verschiedenen Blutarten, der Hauptfrage, die dem Gerichtschemiker gestellt $\mathrm{zu}$ werden pflegt. Die völlig sichere Beantwortung dieser Frage ist jedoch erst durch die biologischen Methoden möglich geworden; wir haben daher der Beschreibung dieser besondere Sorgfalt widmen zu sollen geglaubt, zumal da sie nicht nur für den Mediziner, sondern auch für den Gerichtschemiker große Bedeutung erlangt hat und eine zusammenfassende und ausführliche Darstellung dieses Gebietes für diesen Zweck bisher noch nicht erfolgt ist. 
Ein Anhang behandelt die Brandstiftungen, aber auch diese nur soweit, als der Gerichtschemiker dabei zu Rate gezogen werden kann.

Hier tritt die Photographie vollständig zurück, wenn man nicht auch die photographischen Aufnahmen des Tatortes mit in den Kreis der Betrachtung ziehen will; das fällt aber mehr in das Gebiet der die Untersuchung führenden Organe, der Polizei und des Gerichts. Wir haben uns daher davon vollständig zurückgehalten, zumal ausgezeichnete und umfassende Werke, die diesen Gegenstand erschöpfend behandeln, existieren.

Wenn nun auch die folgenden Ausführungen in erster Linie für Chemiker, aber auch für Mediziner und Pharmazeuten, die vor allen in den einschlägigen Fragen als Sachverständige herangezogen zu werden pflegen, geschrieben sind, so haben wir doch geglaubt, uns jedem Gebildeten verständlich ausdrücken zu sollen. Wir haben dabei in erster Linie an die Juristen gedacht, sowohl an den die Untersuchung führenden, wie den erkennenden Richter. Beiden ist eine genaue Kenntnis der für diese Untersuchungen in Betracht kommenden Methoden durchaus unentbehrlich, sei es, daß sie wissen müssen, in welchen Fällen sie den Sachverständigen zu Rate ziehen und welche Fragen sie mit einiger Aussicht auf Erfolg stellen können, sei es, daß sie in den Stand gesetzt werden, sich über den Wert oder Unwert eines erstatteten Gutachtens ein einigermaßen selbständiges Urteil zu bilden. Dasselbe gilt für den eine Kriminaluntersuchung führenden Polizeibeamten.

Auf keinem Gebiete der gerichtlichen Expertise kann so viel gesündigt und gefehlt werden, wie bei der Untersuchung von Urkundenfälschungen und dergleichen. Wir sehen dabei vom Schriftsachverständigen vollständig ab, auch der gerichtliche Photograph ist, wie jener, manchen Gefahren ausgesetzt und kann, wenn er kritiklos arbeitet, durch seine Methoden etwas hervorheben, verstärken oder gar erzeugen, was in Wirklichkeit anders ist oder gar nicht existiert.

Die Gefahr ist hier um so größer, weil der Laie zu der Ansicht geneigt ist, die Photographie könne nicht lügen und was ein Photogramm zeige, müsse in Wirklichkeit auch vorhanden sein. Wer die folgenden Ausführungen mit Aufmerksamkeit liest, wird das Irrige dieser Ansicht bald erkennen und vielmehr zu 
der Uberzeugung kommen, daß, wie bei jedem SachverständigenGutachten, so besonders, wenn photographische Methoden mitbenutzt wurden, die sorgfältigste Kritik am Platze ist.

Diese Kritik wird in erster Linie von anderen Sachverständigen zu üben sein, in letzter Linie ist aber doch der erkennende Richter der ausschlaggebende Teil und es ist sehr zu wünschen, daß er sich nicht nur auf die Gutachten der Sachverständigen und deren gegenseitige Kritik verlasse, auf die nach seiner Meinung etwa größere Sachkenntnis oder Glaubwürdigkeit des einen oder des anderen, die vielleicht nur auf der größeren Gewandtheit in dem Auftreten vor Gericht beruht, sondern daß er sich ein eigenes Urteil über die angewandten Verfahren zu bilden versuche.

Endlich wird auch der Verteidiger, zumal wenn er von der Unschuld seines Klienten überzeugt ist, ein Interesse haben, daß ein etwa ungünstig ausgefallenes Gutachten auf seine wirkliche Tragweite geprüft werde. Auch hier werden sich die nötigen Handhaben nur demjenigen bieten, der auch den technischen Ausführungen des Sachverständigen folgen kann.

Aus diesen Gründen haben wir uns einer allgemein verständlichen Ausdrucksweise und Fassung zu befleißigen gesucht, selbst auf die Gefahr hin, dem chemischen Experten, für dessen Gebrauch das Buch in erster Linie bestimmt ist, etwas Altbekanntes und Selbstverständliches zu sagen; in dieser Beziehung haben wir daher zumal den Chemiker um Nachsicht zu bitten.

Hamburg, im Dezember 1905.

Die Verfasser. 


\section{INHALTSVERZEICHNIS.}

Inhaltsverzeichnis . . . . . . . . . . . . . . . IX

Einleitung . . . . . . . . . . . . . . . 1

Urkundentälschung . . . . . . . . . . . . . . . . . . . . . . 6

Sind Schriftzüge mit demselben Material hergestellt? . . . . 9

Tintenschrift . . . . . . . . . . . . . . . . . . . 9

Mikroskopische Prüfung . . . . . . . . . . . . . . . . . . . 32

Aufnahme in Originalgröße oder schwacher Vergrößerung im

durchfallenden Lichte . . . . . . . . . . . . . . . . . . 39

Aufnahme in starker Vergrößerung im durchfallenden Lichte $\mathbf{4 0}$

Aufnahme mit Planaren . . . . . . . . . . . . . . . . . . 40

Aufnahme mit Mikroskopobjektiven . . . . . . . . . . . 41

Chemische Prüfung. . . . . . . . . . . . . . . . 61

Verhalten gegen Wasser und Kopierfähigkeit mit Wasser oder Salzsäure . . . . . . . . . . . . . . . . . . . . . . . . 61

Verhalten gegen Chemikalien . . . . . . . . . . . 63

Die sauren Reagenzien . . . . . . . . . . . . . . . . . 64

Die alkalischen Reagenzien . . . . . . . . . . . . . 67

Die Bleichmittel . . . . . . . . . . . . . . . . . . . . . 68

Bleistiftschrift . . . . . . . . . . . . . . . . . . . 70

Sind auf einem Schriftstücke Schriftzeichen entfernt und eventuell an deren Stelle andere gesetzt worden?. .. . 75

Worin bestanden die entfernten Schriftzeichen?. . . . . . 75

Die mechanische Entfernung der Tintenschrift . . . . . . . . . 75

Die chemische Entfernung der Tintenschrift . . . . . . . . . . 81

Die Entfernung der Bleistiftschrift . . . . . . . . . . . . . . 85

Die Wiederherstellung beseitigter Schriftzeichen . . . . . . . . . . 86

Sind Schriftzüge meist derselben Urkunde zu gleicher oder zu verschiedener Zeit geschrieben; wenn das zweite zutrifft, welche sind älter?. . . . . . . . . . . . 94

Tintenschrift . . . . . . . . . . . . . . . 94

Bleistiftschrift. . . . . . . . . . . . . . . . . . 104

Sind Schriftzüge, meist Namensunterschriften, aus freier Hand geschrieben oder sind sie nach einer Vorlage durchgezeichnet oder mechanisch durchgepaust? . . . . 109

Wie lautete eine überkleckste oder sonst unleserlich gemachte Schrift?. . . . . . . . . . . . . . 114

Befinden sich auf einem Papier Schriftzüge von Geheimtinten?.................. . . 122 


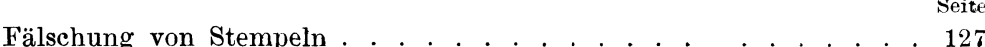

Besonderes .................. . . 133

Beispiel eines Gutachtens . . . . . . . . . . . . . . . . . 145

Blut und Blutflecke . . . . . . . . . . . . . . . . . . . . . . . 153

Photographische Aufnahme von Blutflecken .......... 154

Eigenschaften des Blutes . . . . . . . . . . . . . . . . . 157

Der Nachweis von Blut. . . . . . . . . . . . . . . . . . 161

Unterscheidung von Menschen- und Tierblut . . . . . . . . . . . 182

Die biologische Untersuchung . . . . . . . . . . . . . . 185

Beschreibung des Verfahrens . . . . . . . . . . . . . . . 187

Die Einwirkung giftiger Gase auf Blut . . . . . . . . . . . . . . 196

Sperma und Spermaflecke . . . . . . . . . . . . . . . . 200

Anhang . . . . . . . . . . . . . . . . . . . . . . . . . . 213

Über Brandstiftungen . . . . . . . . . . . . . . . . . . . . . 213

Verordnung, betreffend das Feilhalten von Petroleum, vom 24. Februar 1882 . . . . . . . . . . . . . . . . . . . . 229

Anweisung für die Untersuchung des Petroleums auf seine

Entflammbarkeit mittels des A belschen Petroleumprobers . 230

Hamburgisches Regulativ für die amtliche Kontrolle des im

Gebiete des Petroleumhafens lagernden Petroleums . . . . 237

Namenregister . . . . . . . . . . . . . . . . . . . . . . . 243

Sachregister . . . . . . . . . . . . . . . . 245 